ORIGINAL ARTICLE

\title{
In vivo and in vitro examination of the functional significances of novel lamin gene mutations in heart failure patients
}

\author{
N Sylvius, Z T Bilinska, J P Veinot, A Fidzianska, P M Bolongo, S Poon, P McKeown, R A Davies, \\ K-L Chan, A S L Tang, S Dyack, J Grzybowski, W Ruzyllo, H McBride, F Tesson
}

J Med Genet 2005;42:639-647. doi: 10.1136/jmg.2004.023283

See end of article for authors' affiliations

Correspondence to: Dr F Tesson, University of Ottawa Heart Institute, 40 Ruskin Street, Ottawa, Ontario K1Y 4W7, Canada; ftesson@ ottawaheart.ca

Received 26 May 2004 Revised 31 January 2005 Accepted 4 February 2005

\begin{abstract}
Context: Lamin A/C (LMNA) gene variations have been reported in more than one third of genotyped families with dilated cardiomyopathy (DCM). However, the relationship between LMNA mutation and the development of DCM is poorly understood.

Methods and results: We found that end stage DCM patients carrying LMNA mutations displayed either dramatic ultrastructural changes of the cardiomyocyte nucleus (D192G) or nonspecific changes (R541S). Overexpression of the D192G lamin C dramatically increased the size of intranuclear speckles and reduced their number. This phenotype was only partially reversed by coexpression of the D192G and wild type lamin C. Moreover, the D192G mutation precludes insertion of lamin C into the nuclear envelope when co-transfected with the D192G lamin A. By contrast, the R541S phenotype was entirely reversed by coexpression of the R541S and wild type lamin C. As lamin speckle size is known to be correlated with regulation of transcription, we assessed the SUMO1 distribution pattern in the presence of mutated lamin C and showed that D192G lamin C expression totally disrupts the SUMO1 pattern.

Conclusion: Our in vivo and in vitro results question the relationship of causality between LMNA mutations and the development of heart failure in some DCM patients and therefore, the reliability of genetic counselling. However, LMNA mutations producing speckles result not only in nuclear envelope structural damage, but may also lead to the dysregulation of cellular functions controlled by sumoylation, such as transcription, chromosome organisation, and nuclear trafficking.
\end{abstract}

$\mathrm{D}$ ilated cardiomyopathy (DCM) is characterised by dilatation of cardiac chambers and impaired contraction, leading to heart failure or sudden death. Symptoms are highly variable in severity and age of onset. Penetrance has been found to be incomplete and age related in most families. ${ }^{1-3}$ DCM appears to be a complex genetic disorder, with 16 genes being implicated in autosomal dominant DCM (OMIM \#115200). What is remarkable about DCM is that a variation in one of these genes does not result exclusively in a DCM phenotype. In particular, lamin $\mathrm{A} / \mathrm{C}(L M N A)$ mutations have also been described in at least nine other diseases, including Emery-Dreifuss and other types of muscular dystrophy, lipodystrophy disorders, Charcot-Marie-Tooth disorder type 2, Hutchinson-Gilford progeria syndrome, and restrictive dermopathy (OMIM $\left.\# 150330^{4}\right)$. We calculated from extensive review of the literature that more than one third of reported DCM genotyped families carry a mutation in LMNA. The mechanism by which a mutation in LMNA alters nuclear function and causes disease remains unclear. So far, only two publications have described ultrastructural and immunochemical changes associated with LMNA defects in DCM: ${ }^{5}$ (a) reduced LMNA expression in the myocyte nuclei, and $(b)$ nuclear membrane damage, such as focal disruptions, blebs, and nuclear pore clustering.

In this report, we describe new mutations found through LMNA screening in our DCM population. We determined the effect of $L M N A$ mutations in heart tissue obtained from two patients carrying these novel mutations. In order to understand the cellular mechanisms leading to the observed cardiac phenotypes, we expressed LMNA mutations in a cellular model and performed live cell analysis. We found that overexpression of a specific mutated lamin C influences the spatial organisation of lamin intranuclear speckles. Previous studies have suggested a role for these speckles in controlling gene expression. ${ }^{7}$ We show here that an LMNA mutation is able to disrupt the normal nucleoplasmic distribution of the small ubiquitin-like modifier 1 (SUMO1), which is emerging as a major regulatory protein involved in chromatin organisation and gene expression. ${ }^{8}$

\section{METHODS}

Subjects, heart tissues, and LMNA screening

Written informed consent was obtained from 92 DCM probands and their relatives in accordance with study protocols approved by hospital ethics committees.

Each amplified DNA fragment from LMNA was submitted to both single strand conformational polymorphism electrophoresis ${ }^{9}$ and denaturing high performance liquid chromatography (DHPLC) (Helix, Varian) analysis.

Detailed descriptions of patient and family recruitment, heart tissue collection and processing, and screening methods are provided as supplementary online information at http:// jmg.bmjjournals.com/supplemental/.

\section{Construction of expression plasmids}

Full length lamin C cDNA was obtained by PCR amplification of first strand cDNAs (forward primer 5'-GGAATTCCATGGAGACCCCGTCCCAGCG-3', reverse primer 5'-GCGGATCCTCAGCGGCGGCTACCACTCA-3'). The amplicon was inserted in the multiple cloning sites of both pECFP-Cl (cyan) and

Abbreviations: DCM, dilated cardiomyopathy; SUMO1, small ubiquitin-like modifier 1 
pEYFP-Cl (yellow) vectors (Clontech Laboratories). The cDNA encoding wild type prelamin A, cloned into pcDNA4 (Invitrogen) was kindly provided by Drs Morris and Holt (North East Wales Institute, UK). The vector was transformed in XL competent Escherichia coli. Point mutations found in DCM patients were introduced by using the Transformer site directed mutagenesis kit (Clonetech Laboratories). SUMOI amplified by PCR was fused to pEYFP-C3. Deletion of the five C-terminal residues in the pEYFP:SUMOI construct was achieved by replacing glycine 97 with a stop codon. All clones were systematically sequenced.

\section{Cell culture and transfection}

COS7 cells (ATCC) were cultured on glass coverslips in Dulbecco's modified Eagle's medium glutamax, supplemented with $10 \%$ fetal bovine serum and $1 \%$ L-glutamine. The transfection of fusion protein constructs was made using $0.5 \mu \mathrm{g}$ of expression vectors and Lipofectamine 2000 (Life Technologies). Cells were grown for 17-21 hours. Both cyclohexamide $(28 \mu \mathrm{g} / \mathrm{ml})$ and Hoechst 33258 Dye $(10 \mu \mathrm{g} /$ $\mathrm{ml}$ ) were added for 30 minutes prior to microscopic observation to halt transcription and visualise chromosomes, respectively. Excitation wavelengths were $434 \mathrm{~nm}$ for CFP:lamin C, $514 \mathrm{~nm}$ for YFP:lamin C, YFP:SUMOl, and

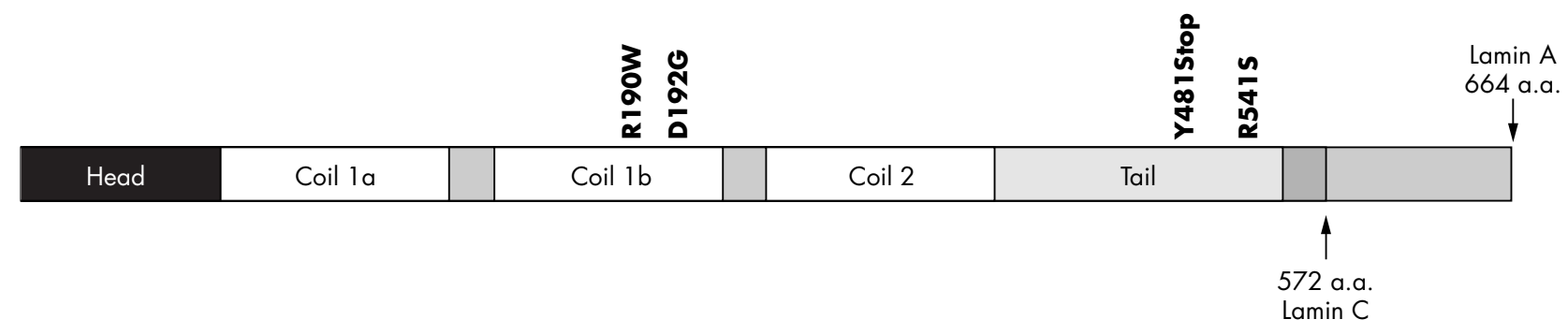

Y481Stop

।

॥

III

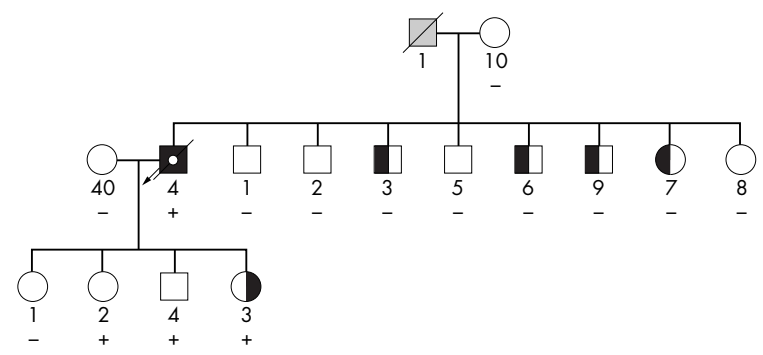

D192G

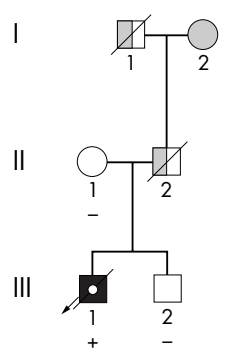

R190W
R541S

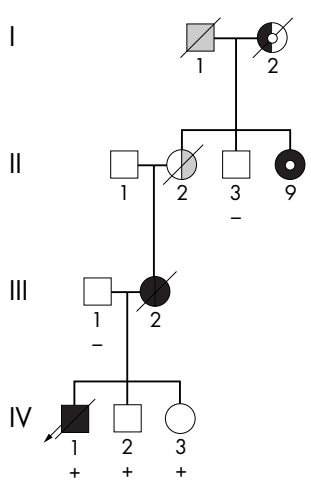

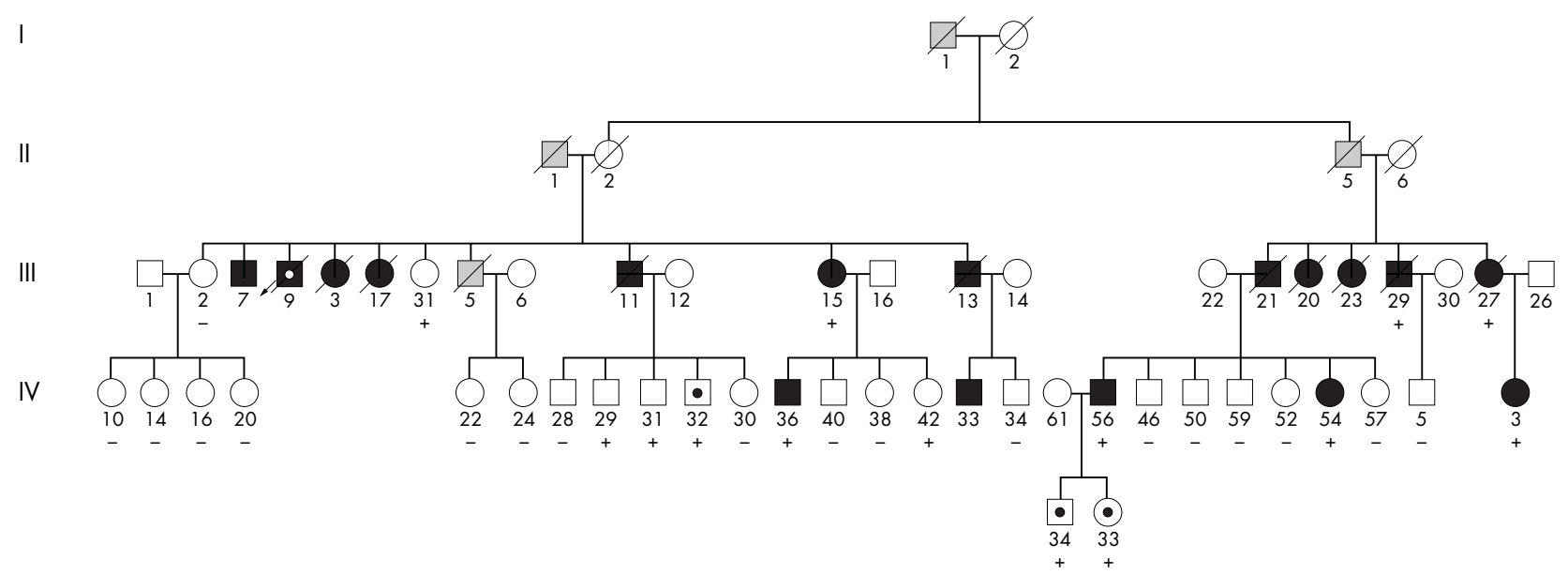

Figure 1 Pedigree of the families with LMNA mutations and location of the identified mutation on a schema of LMNA primary sequence. Arrow indicates proband; black filled symbol, dilated cardiomyopathy; open symbol, asymptomatic; grey filled symbol, no clinical data; left half filled symbol (black), left ventricular dilatation; right half filled symbol (black), right bundle branch block; left half filled symbol (grey), heart failure; right half filled symbol (grey), sudden death; black filled dot within symbol, unknown dilated cardiomyopathy status; grey filled dot within symbol, muscular dystrophy; diagonal lines, death; + or - sign, presence or absence of mutations. 
YFP-SUMOI $\Delta$ C5, $350 \mathrm{~nm}$ for Hoechst 33258 dye, and $575 \mathrm{~nm}$ for secondary antibodies.

\section{Timelapse microscopy}

Glass coverslips were mounted in live cell chambers in regular growth media supplemented with $20 \mathrm{mmol} / \mathrm{l}$ HEPES $(\mathrm{pH} 7.4)$, visualised at $100 \times$ magnification in an enclosed Olympus $1 \times 70$ inverted microscope, and processed using TILLvisION software (version 4.0). Videos were taken through a CFP/YFP dual pass filter (Till Photonics, $500 \mathrm{~ms}$ ).

\section{Immunochemistry and immunoblotting}

The primary antibodies used were lamin A/C 131C3 monoclonal antibody (Chemicon International), lamin A 133A2 monoclonal antibody (Abcam), emerin G45 monoclonal antibody (Novocastra Laboratories), and SUMO 1/Sentrin polyclonal antibody (Oncogene research product). Detailed description is provided as supplementary online information at http://jmg.bmjjournals.com/supplemental/.

\section{RESULTS}

\section{LMNA mutations and clinical characteristics of mutations carriers}

In total, 92 DCM probands were screened for variations within LMNA coding sequence and exon-intron boundaries. Four mutations in four unrelated DCM families were found. All mutations were located in highly conserved regions of the gene shared by lamin $\mathrm{A}$ and $\mathrm{C}$ isoforms. Expressed phenotypes in the 22 mutation carriers were highly variable between families and within families. There were 10 symptomatic mutation carriers, while four adult mutation carriers (age range 23-43 years) and four children (age range 6-14 years) were asymptomatic (clinical characteristics of LMNA mutation carriers are provided as supplementary online information at http://jmg.bmjjournals.com/supplemental/). Symptomatic mutation carriers were characterised by poor prognosis. In total, of 22 symptomatic family members, 5 patients received heart transplant and 12 died from DCM.

The Rl90W mutation (C780T transition) was found in a large family from the UK with autosomal dominant inheritance (fig 1). Clinical features for some of the family members have been detailed in a previous publication. ${ }^{10}$ The D192G mutation (A787G transition) (GenBank accession number AY847595) was found in a Polish family with suspected autosomal dominant transmission (fig 1). The Y481stop mutation (C1656G transversion) (GenBank accession no. AY847596) was found in an unrelated Polish patient (fig 1) and the R541S mutation (C1833A transversion) (GenBank accession no. AY847597) in a Canadian family with suspected autosomal dominant transmission (fig 1).

\section{Pathological studies of hearts from patients with end stage DCM}

Light microscopy of the two available heart samples from patients carrying D192G and R541S LMNA mutations revealed non-specific myocyte damage and interstitial fibrosis (data not shown). Electron micrographs of the heart tissue sections from the D192G LMNA patient demonstrated dramatic morphological alterations, including a complete loss of the nuclear envelope (fig 2A), the accumulation of mitochondria, glycogen and/or lipofuscin in the nucleoplasm, and chromatin disorganisation (fig 2B) in approximately $30 \%$ of nuclei. By contrast, examination of the heart tissue sections from the patient with R541S LMNA revealed only non-specific nuclear membrane alterations (fig 2C), comparable with those found in transplanted DCM patients without LMNA mutation (fig 2D). Immunostaining with mouse antihuman lamin A + C monoclonal antibody showed normal staining of interstitial, vascular, and myocyte nuclei for both the D192G and R541S patient hearts (fig 2E,F,G).

\section{Effects of overexpression of mutated LMNA in COS7 cell model}

To characterise the consequences of LMNA mutations at the cellular level, transient cell transfections were performed on COS7 cells with wild type and/or mutated lamin C mRNA expressed as fusions to the C-terminus of variants of the Aequorea victoria green fluorescent protein (GFP) (lamin CFP). Wild type and mutated lamin C-FP appeared to strictly colocalise with Hoechst 33258 chromosome dye in the nucleus (fig 3A). Wild type lamin C-FP formed distinct intranucleoplasmic foci in most cells. This individualisation of the intranucleolar granules in numerous discrete speckles was previously observed in Swiss $3 \mathrm{~T} 3$ and SW13 cells, ${ }^{11}{ }^{12}$ (fig 3A). Timelapse video fluorescence microscopy indicated that the intranuclear structures were relatively stable (up to 5 hours) and showed no obvious alteration in number, shape, or location over the time period. In a few cells, we observed a smaller number of spots, which were much larger and tended to be round. During transient expression of D192G lamin C$\mathrm{FP}$, the number of cells displaying a few large fluorescent rounded spots was dramatically increased compared with wild type lamin C-FP $(\mathrm{p}<0.0001$; fig $3 \mathrm{~A}, \mathrm{C})$. Indeed, a single giant round spot was present in the nucleus of a mean (SD) $84(6) \%$ of cells. This spot appeared to be stable in shape and
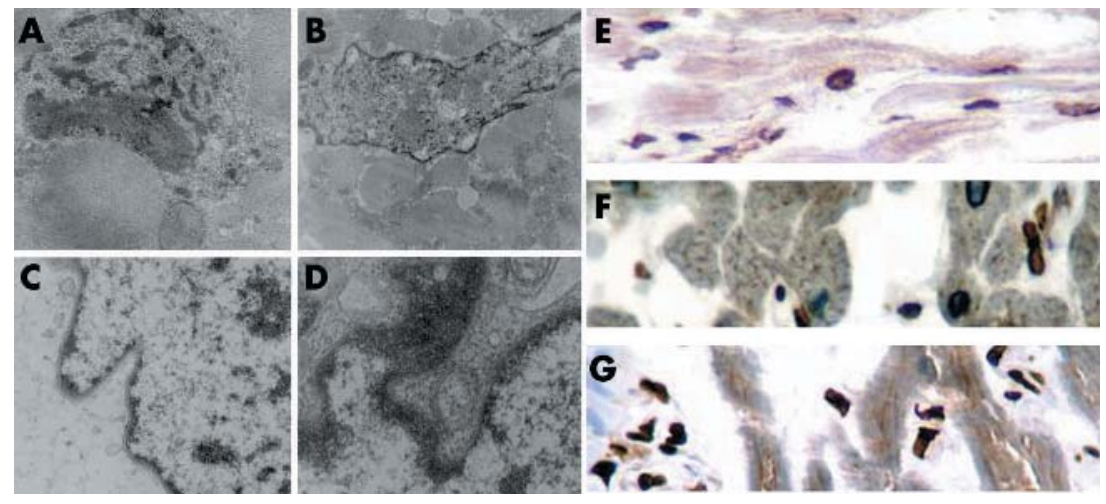

Figure 2 (A-D) Electron micrographs of cardiomyocytes from end stage dilated cardiomyopathy patients with or without LMNA mutation. (A) Total loss of nuclear membrane in DI92G LMNA patient; (B) accumulation of mitochondria and glycogen in the nucleoplasm and chromatin disorganisation in DI92G LMNA patient; (C) non-specific alteration in R541S LMNA patient; (D) non-specific alteration in end stage dilated cardiomyopathy patient with wild type LMNA; $(E-G)$ normal immunostaining with mouse antihuman lamin $A+C$ monoclonal antibody in D192G, R541S, and wild type LMNA patients. (Original magnifications: (A) $\times 20$ 000; (B) $\times 12$ 000; (C) $\times 30000$; (D) $\times 60000$; (E-G) $\times 400)$. 
A
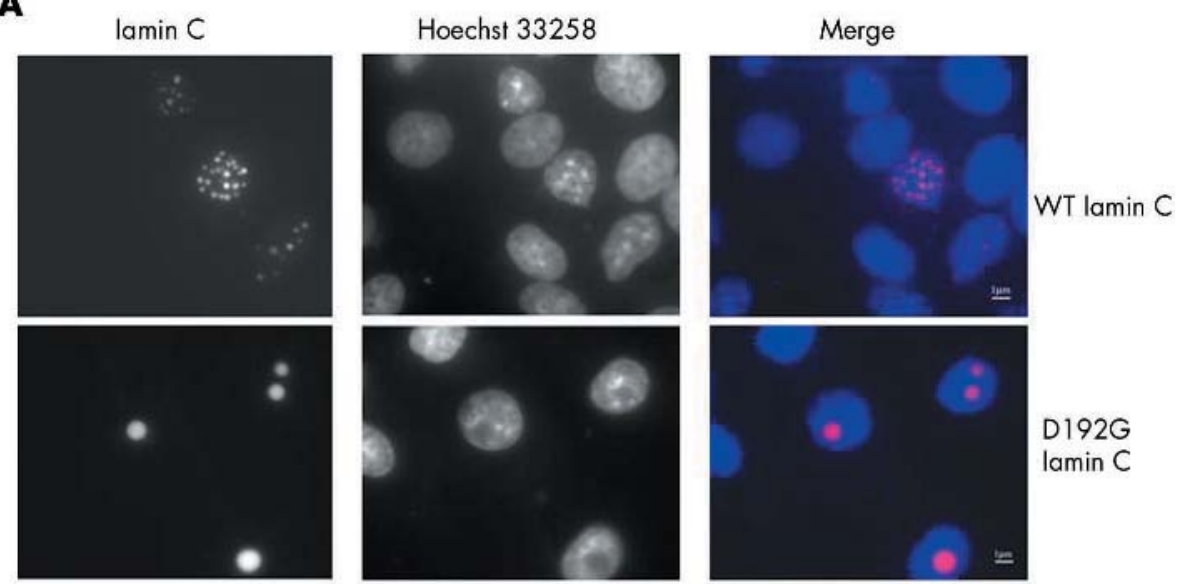

WT lamin C
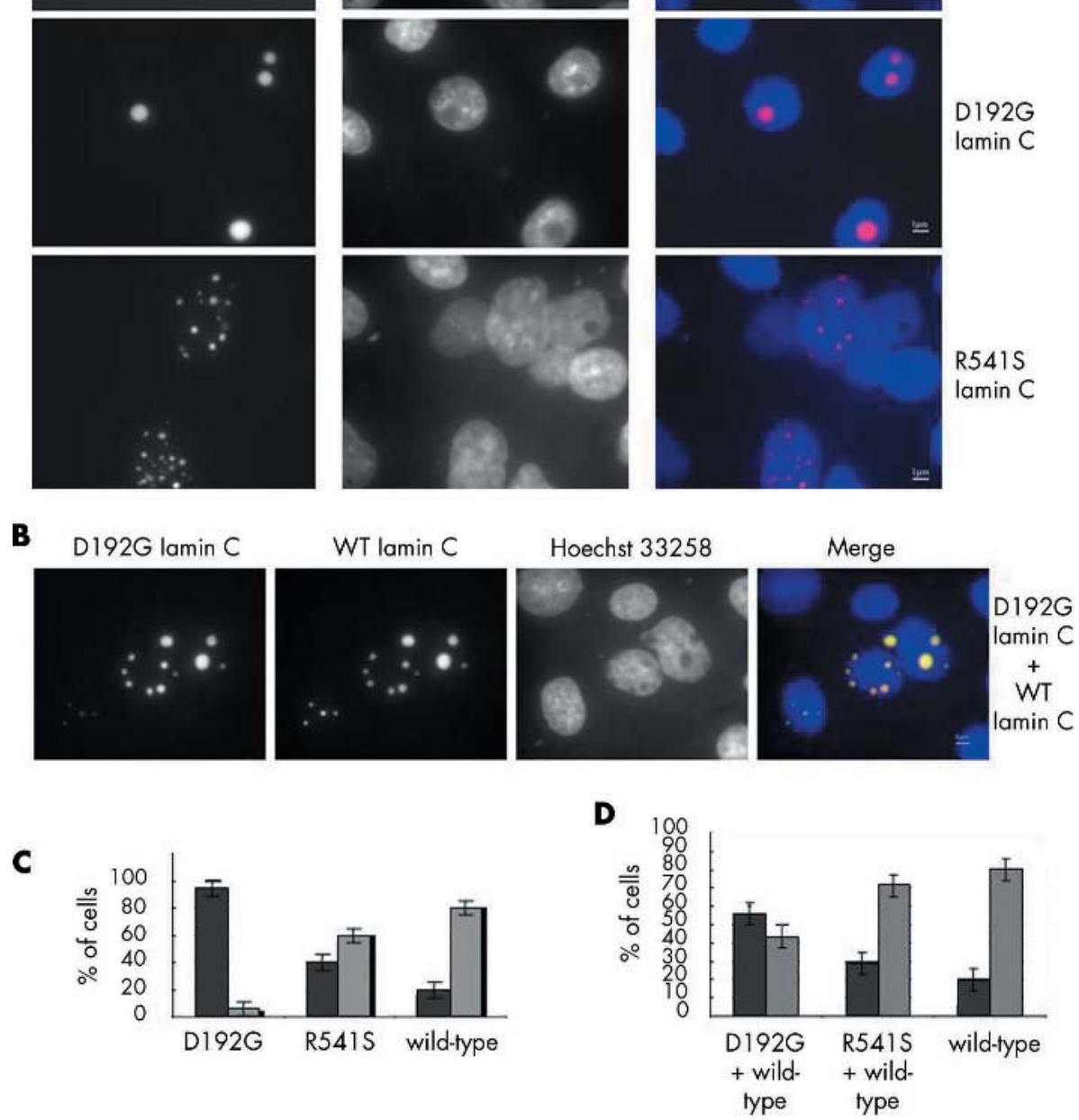

Figure 3 (A) COS7 cells transfected with fluorescently tagged wild type (WT) lamin C, D192G lamin C, and R541S lamin C showing the location of the lamin C (red) within the nucleus visualised by Hoechst 33258 dye (blue). (B) COS7 cells co-transfected with equimolar amounts of fluorescently tagged D192G (red) and wild type lamin C (yellow green) showing their co-location within the nucleus. Ratio of fluorescence was approximately 1. (C, D). Number of spots per transfected cells or cells co-transfected with wild type and mutant lamin C. Data are presented as mean (standard error) calculated from at least three independent experiments. $\chi^{2}$ tests showed significant differences across genotypes $(p<0.0001)$ for single transfection experiments (C). The number of spots was significantly different in cells coexpressing D192G and WT lamin C compared with cells expressing WT lamin C ( $p<0.0001)$, and in cells coexpressing D192G and WT lamin C compared with cells co-expressing R541S and WT lamin C ( $<<0.0001)$. The number of spots was not significantly different in cells co-expressing R541S and WT lamin C compared with cells expressing WT lamin C ( $p>0.1$ ). Black bars, number of cells with $<3$ spots; grey bars, number of cells with $>3$ spots.

location within the nucleus, as indicated by overnight timelapse videos. Transfection efficiency range was 30-60\%. Western blotting quantification of endogenous versus transfected lamin showed that overexpression resulted in a 2.2fold to 15.7-fold increase, with a mean (SD) overexpression of 10.08 (6.0). However, the D192G lamin C-FP typical pattern was obvious in every experiment regardless of the transfection efficiency or the overexpression levels. Importantly, the presence of these giant spots in the D192G lamin C-FP transfected cells could not be explained by a higher rate of expression of the mutated than the wild type lamin C-FP, as the transfection ratio of mutated versus wild type was not different from $1(1.15(0.07))$.
In order to mimic a heterozygous state, we coexpressed wild type and the D192G lamin C. Coexpression only partly restored the wild type phenotype (fig 3B), with approximately $50 \%$ of cells displaying a wild type phenotype (fig 3D). Wild type and mutated lamin C co-distributed in the nucleoplasmic foci (fig 3B). Transient expression of the R541S lamin C-FP led to an intermediate phenotype with fewer and more spherical foci than the wild type phenotype, the number of cells displaying a unique spot being about $40 \%$ (fig 3A, C). Coexpression of wild type and the R541S lamin C completely restored the wild type phenotype (fig 3D). Overexpression of the R190W and Y481stop lamin C did not result in a specific phenotype (data not shown). 
$\mathbf{A}$
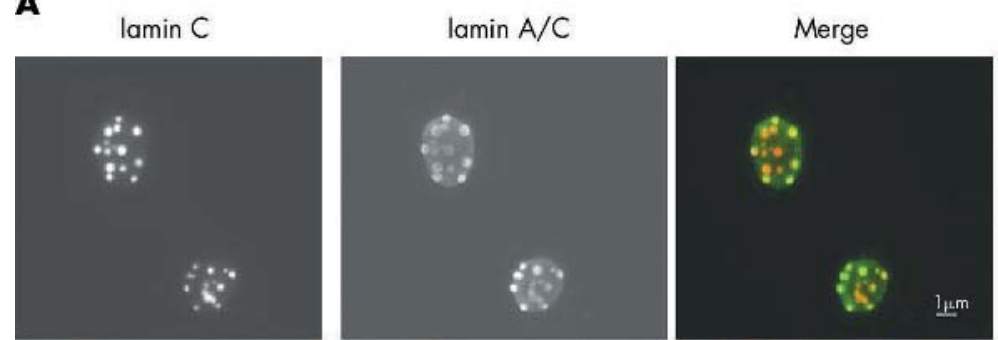

WT lamin C
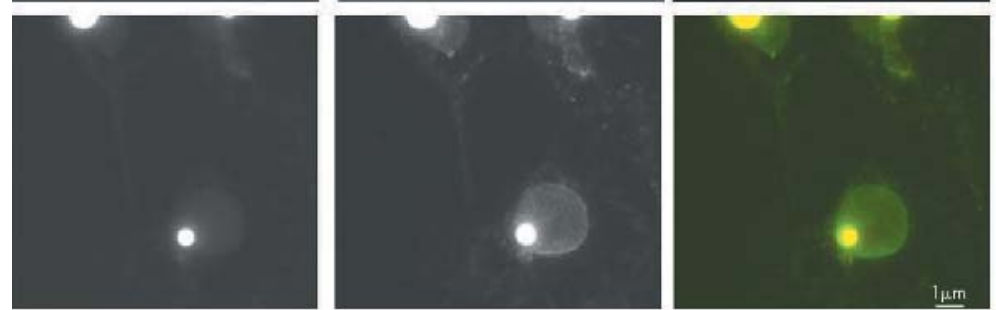

D192G lamin C

B $\quad \operatorname{Lamin} C$
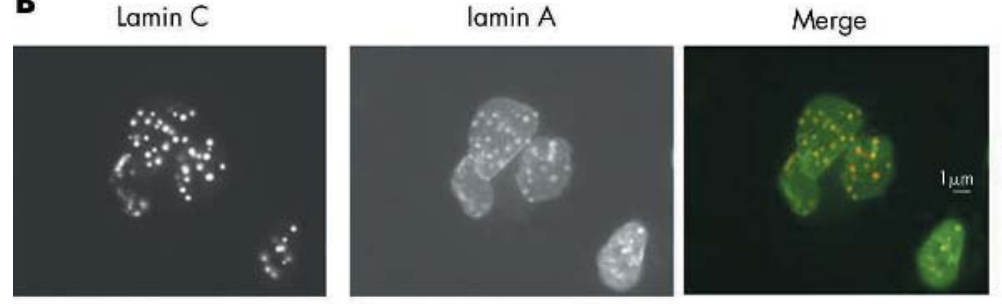

WT lamin C
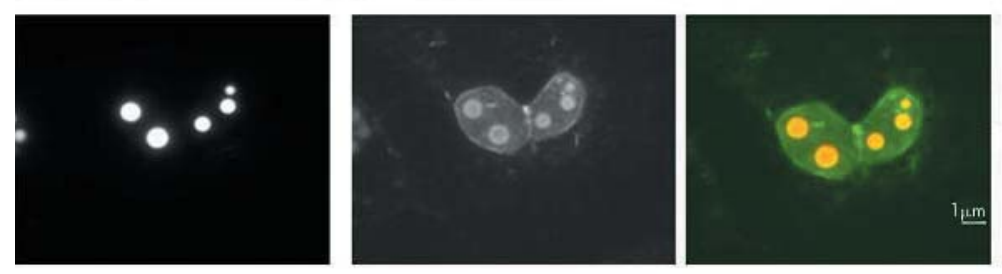

C
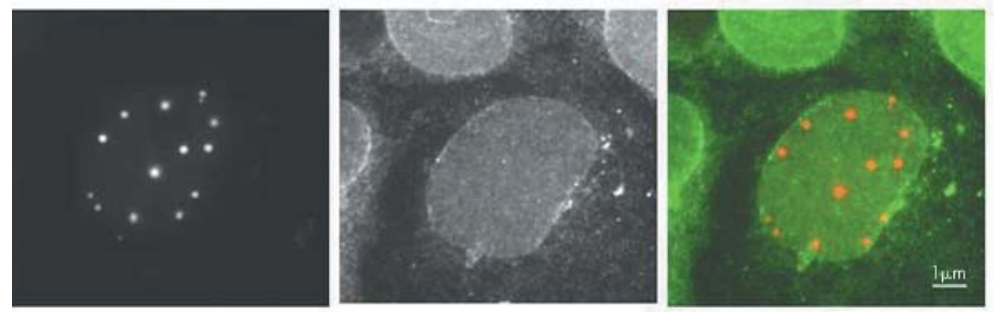

WT lamin C
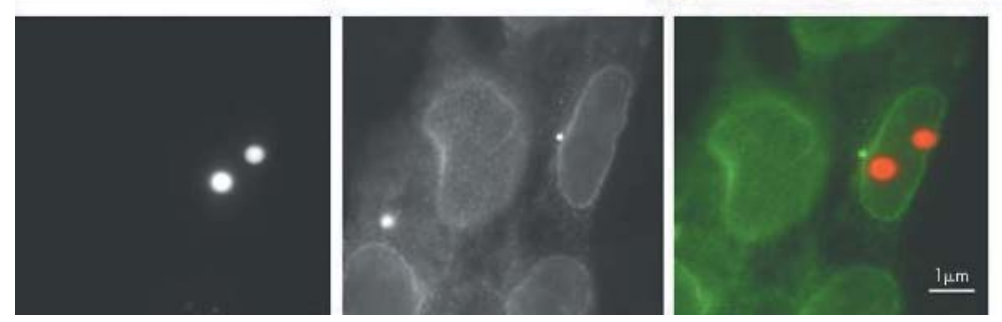

Figure 4 COS7 Cells transfected with fluorescently tagged wild type (WT) or D192G lamin C (red) and labelled with (A) anti-lamin A/C, (B) antilamin A, or (C) anti-emerin antibody (yellow green) and showing that the WT or the D192G lamin C-FP foci did not disturb the distribution of endogenous lamins or emerin on the nuclear rim.

As previously reported for wild type lamin C-FP, ${ }^{11-15}$ wild type or D192G lamin C-FP foci did not disturb the distribution of endogenous lamins or emerin on the nuclear rim as detected by immunofluorescence (fig $4 \mathrm{~A}-\mathrm{C}$ ). However, a readily detectable fraction of the endogenous lamin is recruited at the periphery of the foci (fig $4 \mathrm{~A}, \mathrm{~B}$ ).
It has been suggested that lamin A plays a central role in tethering lamin $\mathrm{C}$ to the nuclear envelope. ${ }^{11-13}$ We tested whether restoration of the stochiometry, through co-transfecting lamin A and C, leads to a relocalisation of lamin C-FP to the nuclear envelope. As shown in fig 5, co-transfection of wild type prelamin A and lamin $\mathrm{C}$ resulted in the redirection 
Lamin C
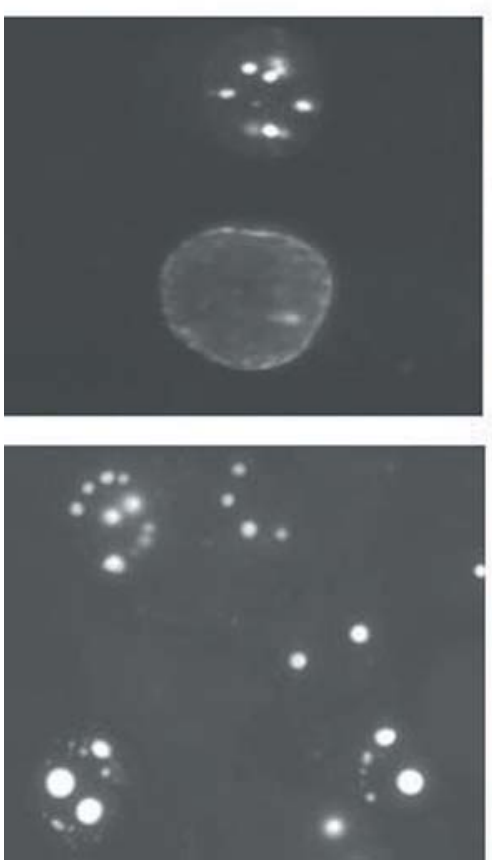

$\operatorname{Lamin} \mathrm{A}$
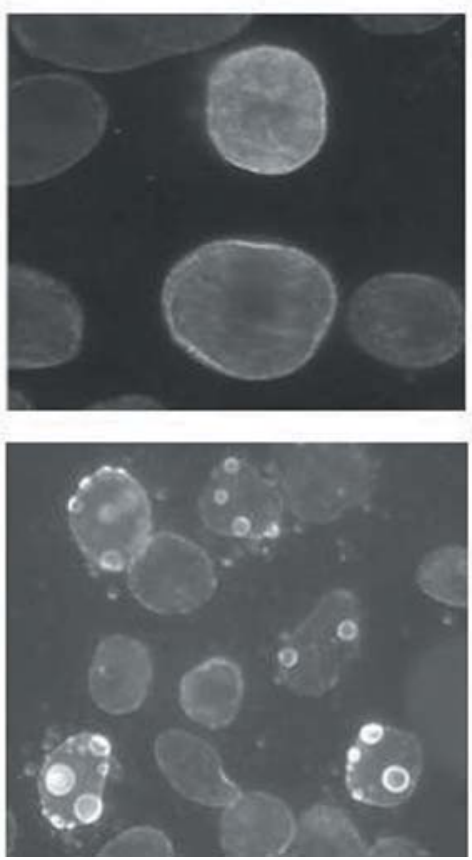

merge

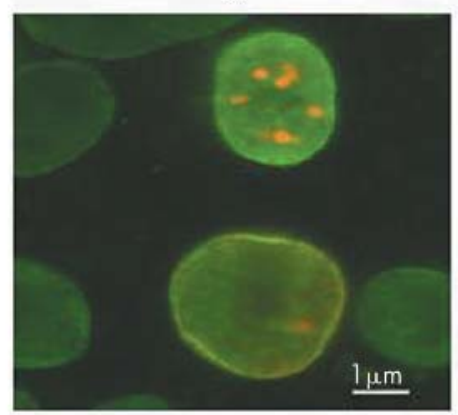

WT lamin A

$+$

WT lamin C

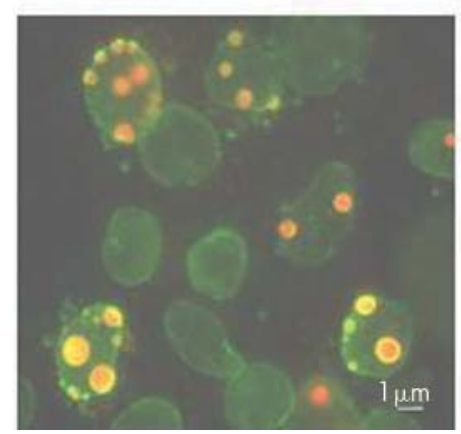

Figure 5 COS7 Cells co-transfected with wild type (WT) prelamin A and fluorescently tagged WT lamin C (red) or D192G prelamin A and fluorescently tagged D192G lamin C (red) and labelled with anti-lamin A antibody (yellow green). The presence of WT lamin A resulted in the retargeting of WT lamin C to the nuclear membrane. D192G lamin C speckles are surrounded by a halo of lamin A, which tends to merge with the nuclear envelope at fusion points. Fluorescently tagged D192G lamin C was not observed in the nuclear envelope.

of lamin C-FP to the nuclear envelope and the reduction of the granular aspect of the nucleoplasm in a significant proportion of the cells. By contrast, this phenomenon was never observed when both the D192G prelamin A and D192G lamin C were co-transfected (fig 5). The D192G lamin C speckles surrounded by the D192G lamin A tend to merge with the nuclear envelope at fusion points where an enrichment of lamin A was observed. However, the D192G lamin $\mathrm{C}$ was never identified in the lamina.

\section{Effect of the D192G LMNA mutation on SUMO1 distribution pattern}

Previous studies have proposed a role for internal lamin speckles in controlling gene expression and demonstrated that the reorganisation of lamin speckles into enlarged foci is correlated to inhibition of transcription. ${ }^{7}$ As major cell regulatory systems targeted by sumoylation are regulation of chromatin organisation and gene expression, ${ }^{8}$ we determined whether the normal distribution of SUMOl was conserved in the presence of mutated lamin C. To this end, we co-expressed wild type or mutated lamin $\mathrm{C}$ and SUMOI or SUMOI $\Delta$ C5 in COS7 cells. Western blotting quantification of endogenous versus transfected SUMOl showed that the overexpression was around fivefold. SUMOI $\Delta$ C5 is a mutant form of SUMOI lacking the C-terminal diglycine motif that is essential for covalent modification by SUMOl. ${ }^{16}$ As shown in fig 6A, SUMOI:FP was normally distributed in cell nucleus. ${ }^{17}$ When SUMO1-FP was co-transfected with the D192G lamin C-FP, SUMOI-FP appeared to be recruited and to concentrate in the middle of giant rounded lamin $C$ spots (fig 6A). Coexpression of SUMO1-FP and R541S lamin C-FP resulted in a phenotype indistinguishable from the wild type phenotype (data not shown). By contrast, the SUMOI $\Delta$ C5-FP pattern remained diffuse in presence of both wild type and mutated lamin C (fig 6B). Therefore, the specificity of the dot-like staining of SUMOl-FP in the presence of the D192G lamin C seemed to be dependent on the sumoylation function of SUMOI.

\section{DISCUSSION}

By screening DNA from 92 DCM probands, we found four mutations confirming that LMNA mutations occur in a significant proportion (4\%) of DCM patients. Although it has been suggested ${ }^{18}$ that LMNA mutation carriers with symptomatic DCM are characterised by poor prognosis, more than $18 \%$ of the adult carrier population we studied was unaffected. While the R190W LMNA mutation was previously reported in two related patients in their forties with DCM associated with conduction system defect, ${ }^{5}$ and in four related patients including one with isolated left ventricular noncompaction, ${ }^{19}$ to our knowledge the D192G, Y481stop and R541S mutations have not been reported elsewhere. However, an Y481H LMNA mutation was found to be associated with autosomal dominant limb girdle muscular dystrophy with cardiac conduction block. ${ }^{20}$ Mutations in the Arg541 codon were reported to be associated with both Emery-Dreifuss muscular dystrophy $(\mathrm{R} 541 \mathrm{H})^{20}$ and DCM with conduction defects (R54lC). ${ }^{21}$ Altogether, these results confirm that, despite DCM being considered a monogenic disorder, additional genetic and/or environmental factors contribute to the clinical phenotype.

Previous studies have reported nuclear membrane damage, such as focal disruptions, blebs, and nuclear pore clustering in myocytes from DCM patients with LMNA mutations. ${ }^{56}$ Similar morphological alterations of the nuclear membrane have been reported in different tissues from LMNA mutation carriers who are affected by other diseases, ${ }^{22-24}$ and in animal models. ${ }^{25-27}$ Based on these findings, it was postulated that the cardiomyocytes from the two end stage DCM patients carrying LMNA mutations would also demonstrate indications of nuclear membrane damage. Surprisingly, while heart tissue sections from the D192G LMNA carrier revealed 
A

SUMO1
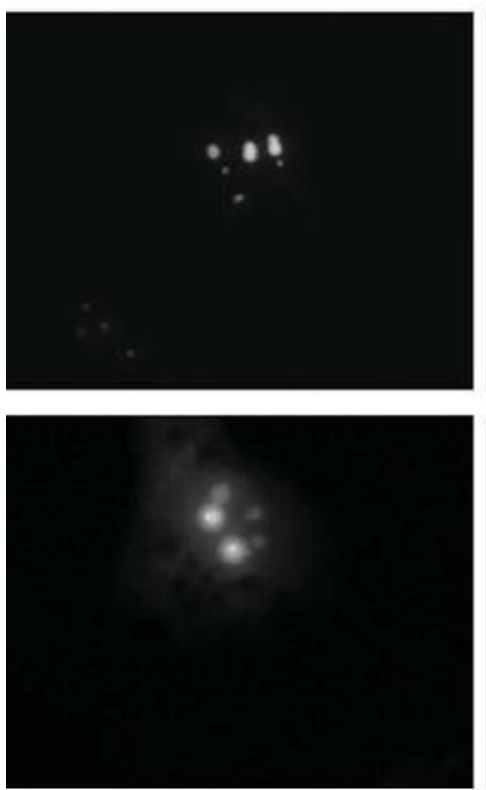

B
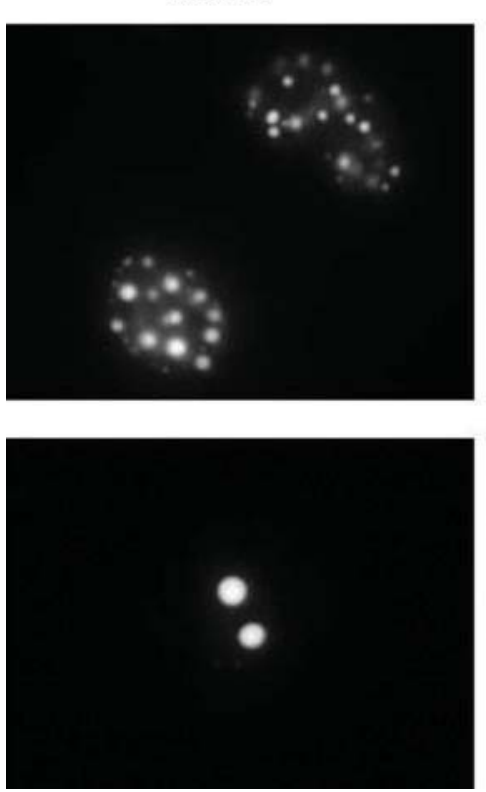

Lamin C
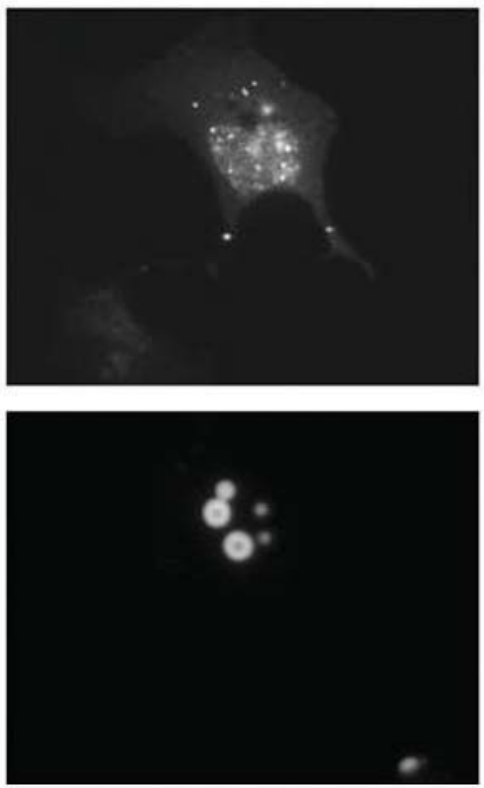

SUMO1 $\triangle \mathrm{C} 5$
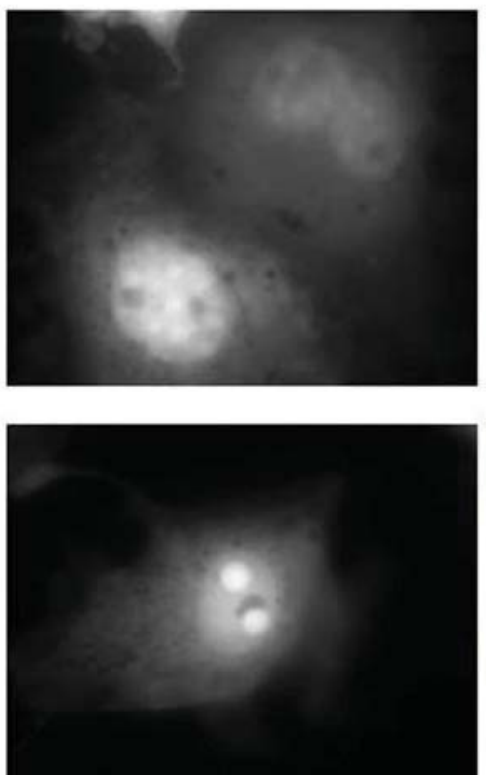

Merge

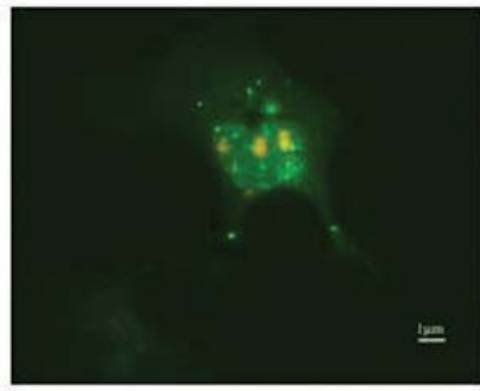

WT Lamin C

$+$

SUMO1

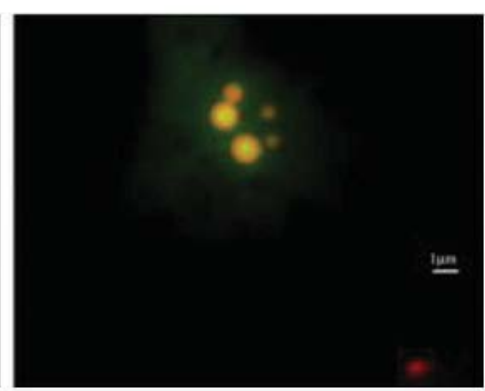

D192G Lamin C $+$ SUMOI

WT Lamin C

SUMO1 $\triangle C 5$

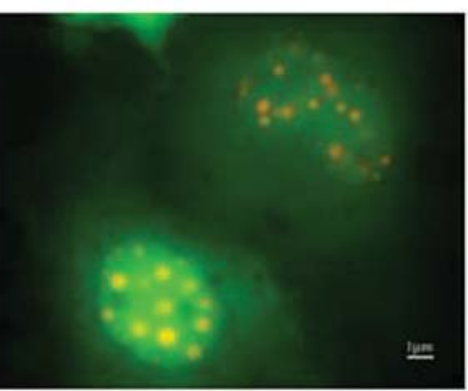

Figure 6 COS7 Cells co-transfected with fluorescently tagged lamin C (red) and (A) SUMO1 or (B) SUMO1 $\triangle C 5$ (yellow green). (A) Presence of wild type lamin C did not alter SUMO1 distribution (small dots within and nearby the nucleus), while co-transfection with D192G lamin C shows recruitment of SUMO1 within the lamin C spots. (B) No difference is observed in the localisation of SUMO1 $\triangle$ C5 in cell transfected with wild type lamin C or D192G lamin.

marked nuclear abnormalities, cardiomyocytes from the patient carrying the R541S mutation presented only modest and non-specific nuclear membrane alterations. It is clear from these observations that direct correlations between any of the morphological changes and the mutations cannot be made.

In order to unravel the observed cardiac phenotypes, we expressed LMNA mutations in a cellular model and performed live cell analysis. While both phenotypes induced by overexpression of the R190W and Y481stop were indistinguishable from the wild type, overexpression of the D192G lamin $\mathrm{C}$ induced a reorganisation of the lamin speckles into a unique and giant round spot in the vast majority of cells, despite expression level of the D192G lamin C being similar to the wild type. When the R541S lamin C was expressed, the phenotype was intermediate with fewer spherical foci than the wild type phenotype. LMNA mutations resulting in punctuate distribution across the nuclear surface of lamin $\mathrm{A}$ and $\mathrm{C}$ have been previously reported in vitro, ${ }^{28} 29$ and in patient fibroblasts. ${ }^{14}{ }^{30}$

To mimic a heterozygous state, we co-transfected the mutated lamin $\mathrm{C}$ with the wild type, and demonstrated that the rearrangement of lamin speckles was partially (D192G) or fully (R541S) reversed. The rescue of the wild type phenotype for R541S may explain why the patient carrying the R541S heterozygous mutation displays non-specific 
cardiac ultrastructural changes. It cannot be excluded that this patient carries a mutation in another DCM gene. By contrast, the discrete giant lamin speckles seen with the D192G lamin C persisted even in the presence of D192G lamin A, suggesting that those mutants are assembly incompetent. The lamin A tended to form a halo around the D192G lamin C speckles, which merged with the nuclear envelope at lamin A enrichment points. This lamin A halo was previously observed when cells were transfected with the N195K, E358K, and M371K mutant lamin A alone; ${ }^{29}$ here we show that the mutant lamin $\mathrm{C}$ is trapped inside. As a consequence of compromised lamina integrity observed in vitro, the nuclear envelope may become more fragile. This may result in the ultrastructural damage of the nuclear envelope, including the loss of the nuclear membrane in the heart tissue of patients carrying such mutations.

Synchronous and reversible formation of enlarged and rounded foci of lamin $\mathrm{A} / \mathrm{C}$ have been described following the inhibition of transcription. ${ }^{7}$ Conversely, disruption of lamin speckles leads to a downregulation of the transcription. ${ }^{7}$ It was speculated that lamin speckles are part of a dynamic structure that can be rapidly modulated by specific signalling events to spatially organise RNA polymerase II transcription. ${ }^{7}$ Given these data and our results, the speckle formation induced by mutated lamin A/C may result in "freezing" of the cells in a transient and/or a pathological phase that corresponds to a deregulated transcription state.

In vitro and in vivo interactions between A-type lamins and transcription factors have been reported..$^{31}{ }^{32}$ In lamin A/C deficient mice that develop DCM, alterations in nuclear architecture are associated with reduced PPAR $\gamma$ expression, a lack of hypertrophic gene activation, and decreased SREBPl (sterol response element binding protein 1) import via nuclear pores. ${ }^{33}$ On the other hand, lamins $\mathrm{A} / \mathrm{C}$ have been suggested to anchor $(a)$ chromatin, through BAF, which can bind directly to DNA, ${ }^{34}$ and $(b)$ nuclear pores by means of their binding to NUP153. ${ }^{35}$ Clustering of nuclear pores has been frequently reported in patients with LMNA mutations. ${ }^{5636}$ Given those observations, we hypothesised that the effect of LMNA mutations might be mediated by the interference of mutated lamin with the SUMOl pathway. Indeed, reversible covalent modification of proteins with SUMOl is emerging as an important system contributing primarily to regulation of gene expression and chromatin organisation. ${ }^{8}$ RNA polymerase II was shown to be sumoylated in human embryonic kidney-293 (HEK-293) cells. ${ }^{8}$ Furthermore, enzymes involved in the SUMO modification process are components of the nuclear pore complex. ${ }^{37}$ SENP2, a SUMO cleaving protease, interacts with the FG repeat domain of NUP153, the nucleoporin that interacts with the lamins. ${ }^{35}{ }^{37}$ When SUMOI was co-transfected with the D192G lamin C in COS7 cells, all SUMOl conjugated proteins appeared to be recruited and trapped in the middle of giant lamin C spots. Moreover, this abnormal pattern of distribution is dependent on the sumoylation function of SUMO1. As the formation of type A lamin foci was reported for multiple $L M N A$ mutations both in vitro, ${ }^{28} 29$ and in patient fibroblasts, ${ }^{14}{ }^{30}$ this new mechanism by which mutated type A lamin might exert their effects may be common to those mutations. Mutations leading to speckle formation are located all along the $L M N A$ gene and are predicted to modify several amino acids from position 85 to position $530 .{ }^{14} 28-30$ Those mutations were linked to DCM, autosomal dominant Emery-Dreifuss muscular dystrophy, as well as Dunningantype familial lipodystrophy. ${ }^{14}{ }^{28-30}$ By abolishing or reducing the availability of the sumoylated protein pool in the nucleus, those mutations may disturb functions controlled by sumoylation.
In conclusion, we showed an absence of specific phenotype for the R54IS LMNA mutation both in vivo and in vitro. These findings question the supposition that the R54IS LMNA mutation is the primary genetic defect responsible for the development of heart failure in such patients and therefore, raises concerns regarding the reliability of genetic counselling to patients and their families. By contrast, the D192G LMNA mutation is associated in vivo with dramatic morphological changes of the cardiomyocyte nucleus and in vitro with total disorganisation of type A lamins; this lamin mutant trapped SUMOl modified proteins presented in the nucleus. Therefore, our hypothesis is that LMNA mutants leading to the formation of intranuclear speckles induce not only nuclear envelope structural damage, but also the deregulation of cellular functions controlled by sumoylation, such as transcription, chromosome organisation, and protein and RNA nuclear trafficking.

\section{ACKNOWLEDGEMENTS}

These studies would not have been possible without the invaluable assistance of patients and family members. We wish to thank $\mathrm{P}$ Rippstein and W Parks (Ottawa Hospital Department of Pathology) for their excellent histological technical assistance, and from the University of Ottawa Heart Institute, $\mathrm{R}$ Brown for his helpful technical advice, $\mathrm{K}$ Williams for her assistance with statistical calculations, R Zunino for his excellent technical assistance, and $\mathrm{K}$ Adamo for her critical review of the manuscript. We also thank Drs G E Morris and I Holt (North East Wales Institute, UK) for the gift of prelamin A cDNA.

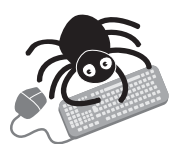

Supplementary information can be viewed on the JMG website at http://www.jmedgenet.com/supplemental.

\section{Authors' affiliations}

N Sylvius, P M Bolongo, S Poon, F Tesson, Laboratory of Genetics of Cardiac Diseases, University of Ottawa Heart Institute, Ottawa, ON, Canada

Z T Bilinska, J Grzybowski, W Ruzyllo, National Institute of Cardiology, Warsaw, Poland

J P Veinot, Department of Pathology and Laboratory Medicine, University of Ottawa, Anatomical Pathology, Ottawa Hospital, Ottawa, ON, Canada

A Fidzianska, Polish Academy of Sciences, Warsaw, Poland

P McKeown, Department of Medicine, Queen's University of Belfast, Belfast, UK

R A Davies, K-L Chan, A S L Tang, Division of Cardiology, University of OHtawa Heart Institute, Ottawa, ON, Canada

S Dyack, Department of Pediatrics, Dalhousie University, Halifax, NS, Canada

H McBride, Lipoproteins and Atherosclerosis Unit, University of Ottawa Heart Institute, Ottawa, ON, Canada

This research was supported by Canadian Institutes for Health Research operating grants 38054 and 65152, and by Heart and Stroke Foundation grant NA 5101 awarded to F Tesson. Polish patients and families were studied with support of grant no. 0010/P05B/98/14 from the Polish Committee for Scientific Research. N Sylvius is the recipient of the fellowship awarded by the Heart and Stroke Foundation of Ontario Program grant 5275 .

Competing interests: none declared

\section{REFERENCES}

1 Mangin L, Charron P, Tesson F, Mallet A, Dubourg O, Desnos M, Benaische A, Gayet C, Gibelin P, Davy JM, Bonnet J, Sidi D, Schwartz K, Komajda M. Familial dilated cardiomyopathy: clinical features in French families. Eur J Heart Fail 1999;1:353-61

2 Mestroni L, Rocco C, Gregori D, Sinagra G, Di Lenarda A, Miocic S, Vatta M, Pinamonti B, Muntoni F, Caforio AL, McKenna WJ, Falaschi A, Giacca M, Camerini. Familial dilated cardiomyopathy: evidence for genetic and phenotypic heterogeneity. Heart Muscle Disease Study Group. J Am Coll Cardiol 1999;34:181-90. 
3 Sylvius N, Tesson F, Gayet C, Charron P, Benaiche A, Peuchmaurd M Duboscq-Bidot L, Feingold J, Beckmann JS, Bouchier C, Komaja M. A new locus for autosomal dominant dilated cardiomyopathy identified on chromosome 6q12-q16. Am J Hum Genet 2001;68:241-6.

4 Navarro CL, De Sandre-Giovannoli A, Bernard R, Boccaccio I, Boyer A, Genevieve D, Hadj-Rabia S, Gaudy-Marqueste C, Smitt HS, Vabres P, Faivre L, Verloes A, Van Essen T, Flori E, Hennekam R, Beemer FA, Laurent N, Le Merrer M, Cau P, Levy N. Lamin A and ZMPSTE24 (FACE-1) defects cause nuclear disorganization and identify restrictive dermopathy as a lethal neonatal laminopathy. Hum Mol Genet 2004;13:2493-503.

5 Arbustini E, Pilotto A, Repetto A, Grasso M, Negri A, Diegoli M, Campana C, Scelsi L, Baldini E, Gavazzi A, Tavazzi L. Autosomal dominant dilated cardiomyopathy with atrioventricular block: a lamin A/C defect-related disease. J Am Coll Cardiol 2002;39:981-90.

6 Verga L, Concardi M, Pilotto A, Bellini O, Pasotti M, Repetto A, Tavazzi L, Arbustini $E$. Loss of lamin A/C expression revealed by immuno-electron microscopy in dilated cardiomyopathy with atrioventricular block caused by LMNA gene defects. Virchows Arch 2003;443:664-71

7 Kumaran RI, Muralikrishna B, Parnaik VK. Lamin A/C speckles mediate spatial organization of splicing factor compartments and RNA polymerase II transcription. J Cell Biol 2002;159:783-93.

8 Zhao Y, Kwon SW, Anselmo A, Kaur K, White MA. Broad spectrum identification of cellular small ubiquitin-related modifier (SUMO) substrate proteins. J Biol Chem 2004;279:20999-1002.

9 Tesson F, Sylvius N, Pilotto A, Dubosq-Bidot L, Peuchmaurd M, Bouchier C, Benaiche A, Mangin L, Charron P, Gavazzi A, Tavazzi L, Arbustini E, Komaja M. Epidemiology of desmin and cardiac actin gene mutations in a european population of dilated cardiomyopathy. Eur Heart $J$ 2000;21:1872-6.

10 MacLennan BA, Tsoi EY, Maguire C, Adgey AA. Familial idiopathic congestive cardiomyopathy in three generations: a family study with eight affected members. Q J Med 1987;63:335-47.

11 Pugh GE, Coates PJ, Lane EB, Raymond Y, Quinlan RA. Distinct nuclear assembly pathways for lamins $A$ and $C$ lead to their increase during quiescence in Swiss 3T3 cells. J Cell Sci 1997; 110:2483-93.

12 Vaughan A, Alvarez-Reyes M, Bridger JM, Broers JL, Ramaekers FC, Wehnert M, Morris GE, Whitfield WGF, Hutchison CJ. Both emerin and lamin $\mathrm{C}$ depend on lamin A for localization at the nuclear envelope. J Cell Sci 2001;114:2577-90.

13 Muralikrishna B, Dhawan J, Rangaraj N, Parnaik VK. Distinct changes in intranuclear lamin $\mathrm{A} / \mathrm{C}$ organization during myoblast differentiation. J Cell Sci 2001;114:4001-11.

14 Muchir A, Medioni J, Laluc M, Massart C, Arimura T, van der Kooi AJ Desguerre I, Mayer M, Ferrer X, Briault S, Hirano M, Worman HJ, Mallet A, Wehnert M, Schwartz K, Bonne G. Nuclear envelope alterations in fibroblasts from patients with muscular dystrophy, cardiomyopathy, and partial lipodystrophy carrying lamin A/C gene mutations. Muscle Nerve 2004:30:444-50.

15 Reichart B, Klafke R, Dreger C, Kruger E, Motsch I, Ewald A, Schafer J, Reichmann H, Muller CR, Dabauvalle MC. Expression and localization of nuclear proteins in autosomal-dominant Emery-Dreifuss muscular dystrophy with LMNA R377H mutation. BMC Cell Biol 2004:5:12.

16 Johnson ES, Schwienhorst I, Dohmen RJ, Blobel G. The ubiquitin-like protein $\mathrm{Sm}+3 p$ is activated for conjugation to other proteins by an Aos $1 \mathrm{p} / \mathrm{Uba} 2 \mathrm{p}$ heterodimer. EMBO J 1997;16:5509-19.

17 Saitoh $\mathrm{H}$, Hinchey J. Functional heterogeneity of small ubiquitin-related protein modifiers SUMO-1 versus SUMO-2/3. J Biol Chem 2000;275:6252-8.

18 Taylor MR, Fain PR, Sinagra G, Robinson ML, Robertson AD, Carniel E, Di Lenarda A, Bohlmeyer TJ, Ferguson DA, Brodsky GL, Boucek MM, Lascor J, Moss AC, Li WL, Stetler GL, Muntoni F, Bristow MR, Mestroni L. Natural history of dilated cardiomyopathy due to lamin A/C gene mutations. J Am Coll Cardiol 2003;41:771-80.

19 Hermida-Prieto M, Monserrat L, Castro-Beiras A, Laredo R, Soler R, Peteiro J Rodriguez E, Bouzas B, Alvarez N, Muniz J, Crespo-Leiro M. Familial dilated cardiomyopathy and isolated left ventricular noncompaction associated with lamin A/C gene mutations. Am J Cardiol 2004;94:50-4.
20 Kitaguchi T, Matsubara S, Sato M, Miyamoto K, Hirai S, Schwartz K, Bonne $G$. A missense mutation in the exon 8 of lamin $A / C$ gene in a Japanese case of autosomal dominant limb-girdle muscular dystrophy and cardiac conduction block. Neuromuscul Disord 2001;11:542-6.

21 Forissier JF, Bonne G, Bouchier C, Duboscq-Bidot L, Richard P, Wisnewski C, Briault S, Moraine C, Dubourg O, Schwartz K, Komajda M. Apical left ventricular aneurysm without atrio-ventricular block due to a lamin $\mathrm{A} / \mathrm{C}$ gene mutation. Eur J Heart Fail 2003;5:821-5.

22 Fidzianska A, Toniolo D, Hausmanowa-Petrusewicz I. Ultrastructural abnormality of sarcolemmal nuclei in Emery-Dreifuss muscular dystrophy (EDMD). J Neurol Sci 1998;159:88-93

23 Vigouroux C, Auclair M, Dubosclard E, Pouchelet M, Capeau J, Courvalin JC, Buendia B. Nuclear envelope disorganization in fibroblasts from lipodystrophic patients with heterozygous R482Q/W mutations in the lamin A/C gene. J Cell Sci 2001;114:4459-68.

24 Novelli G, Muchir A, Sangiuolo F, Helbling-Leclerc A, D'Apice MR, Massart C, Capon F, Sbraccia P, Federici M, Lauro R, Tudisco C, Pallotta R, Scarano G, Dallapiccola B, Merlini L, Bonne G. Mandibuloacral dysplasia is caused by a mutation in LMNA-encoding lamin A/C. Am J Hum Genet 2002;71:426-31.

25 Lenz-Bohme B, Wismar J, Fuchs S, Reifegerste R, Buchner E, Betz H, Schmitt B. Insertional mutation of the Drosophila nuclear lamin $\mathrm{Dm0}$ gene results in defective nuclear envelopes, clustering of nuclear pore complexes, and accumulation of annulate lamellae. J Cell Biol 1997;137:1001-16.

26 Sullivan T, Escalante-Alcalde D, Bhatt H, Anver M, Bhat N, Nagashima K, Stewart CL, Burke B. Loss of A-type lamin expression compromises nuclear envelope integrity leading to muscular dystrophy. J Cell Biol 1999;147:913-20.

27 Mounkes LC, Kozlov S, Hernandez L, Sullivan T, Stewart CL. A progeroid syndrome in mice is caused by defects in A-type lamins. Nature 2003:423:298-301.

28 Raharjo WH, Enarson P, Sullivan T, Stewart CL, Burke B. Nuclear envelope defects associated with LMNA mutations cause dilated cardiomyopathy and Emery-Dreifuss muscular dystrophy. J Cell Sci 2001;114:4447-57.

29 Ostlund C, Bonne G, Schwartz K, Worman HJ. Properties of lamin A mutants found in Emery-Dreifuss muscular dystrophy, cardiomyopathy and Dunnigantype partial lipodystrophy. J Cell Sci 2001;114:4435-45.

30 Capanni C, Cenni V, Mattioli E, Sabatelli P, Ognibene A, Columbaro M, Parnaik VK, Wehnert M, Maraldi NM, Squarzoni S, Lattanzi G. Failure of lamin A/C to functionally assemble in R482L mutated familial partial lipodystrophy fibroblasts: altered intermolecular interaction with emerin and implications for gene transcription. Exp Cell Res 2003;291:122-34.

31 Dreuillet $C$, Tillit J, Kress $M$, Ernoult-Lange $M$. In vivo and in vitro interaction between human transcription factor MOK2 and nuclear lamin A/C. Nucleic Acids Res 2002;30:4634-42.

32 Lloyd DJ, Trembath RC, Shackleton S. A novel interaction between lamin A and SREBP1: implications for partial lipodystrophy and other laminopathies. Hum Mol Genet 2002;11:769-77.

33 Nikolova V, Leimena C, McMahon AC, Tan JC, Chandar S, Jogia D, Kesteven SH, Michalicek J, Otway R, Verheyen F, Rainer S, Stewart CL, Martin D, Feneley MP, Fatkin D. Defects in nuclear structure and function promote dilated cardiomyopathy in lamin A/C-deficient mice. J Clin Invest 2004;113:357-69.

34 Goldman RD, Gruenbaum Y, Moir RD, Shumaker DK, Spann TP. Nuclear lamins: building blocks of nuclear architecture. Genes Dev 2002; 16:533-47.

35 Smythe C, Jenkins HE, Hutchison CJ. Incorporation of the nuclear pore basket protein nupl53 into nuclear pore structures is dependent upon lamina assembly: evidence from cell-free extracts of Xenopus eggs. EMBO $J$ 2000;19:3918-31.

36 Goldman RD, Shumaker DK, Erdos MR, Eriksson M, Goldman AE, Gordon LB, Gruenbaum Y, Khuon S, Mendez M, Varga R, Collins FS. Accumulation of mutant lamin $A$ causes progressive changes in nuclear architecture in Hutchinson-Gilford progeria syndrome. Proc Natl Acad Sci USA 2004; 10:8963-8

37 Zhang $\mathrm{H}$, Saitoh $\mathrm{H}$, Matunis MJ. Enzymes of the SUMO modification pathway localize to filaments of the nuclear pore complex. Mol Cell Biol 2002;22:6498-508. 